TRANSACTIONS OF THE

AMERICAN MATHEMATICAL SOCIETY

Volume 353, Number 7, Pages 2665-2671

S 0002-9947(01)02720-9

Article electronically published on March 2, 2001

\title{
MULTIPLIER IDEALS OF MONOMIAL IDEALS
}

\author{
J. A. HOWALD
}

\begin{abstract}
In this note we discuss a simple algebraic calculation of the multiplier ideal associated to a monomial ideal in affine $n$-space. We indicate how this result allows one to compute not only the multiplier ideal but also the log canonical threshold of an ideal in terms of its Newton polygon.
\end{abstract}

\section{INTRODUCTION}

Multiplier ideals have become quite important in higher dimensional geometry, because of their strong vanishing properties (cf. [1], [3], [4, [5], 6], 12]). They reflect the singularity of a divisor, ideal sheaf, or metric. It is however fairly difficult to calculate multiplier ideals explicitly, even in the simplest cases: the algebraic definition of the multiplier ideal associated to an arbitrary ideal sheaf $\mathfrak{a}$ requires that we construct a $\log$ resolution of $\mathfrak{a}$ and perform calculations on the resolved space. In this note, we compute the multiplier ideal associated to an arbitrary monomial ideal $\mathfrak{a}$. Like $\mathfrak{a}$, it can be described in combinatorial and linear-algebraic terms.

We begin with some definitions. Let $X$ be a smooth quasiprojective complex algebraic variety. Let $\mathfrak{a} \subset \mathcal{O}_{X}$ be any ideal sheaf. By a log resolution of $\mathfrak{a}$, we mean a proper birational map $f: Y \rightarrow X$ with the property that $Y$ is smooth and $f^{-1}(\mathfrak{a})=\mathcal{O}_{Y}(-E)$, where $E$ is an effective Cartier divisor, and $E+\operatorname{exc}(f)$ has normal crossing support.

Definition 1. Let $\mathfrak{a} \subset \mathcal{O}_{X}$ be an ideal sheaf in $X$, and let $f: Y \rightarrow X$ be a log resolution of $\mathfrak{a}$, with $f^{-1}(\mathfrak{a})=\mathcal{O}_{Y}(-E)$. Let $r>0$ be a rational number. We define the multiplier ideal of $\mathfrak{a}$ with coefficient $r$ to be

$$
\mathcal{J}(r \cdot \mathfrak{a})=f_{*} \mathcal{O}_{Y}\left(K_{Y / X}-\lfloor r E\rfloor\right) .
$$

Here $K_{Y / X}=K_{Y}-f^{*} K_{X}$ is the relative canonical bundle, and $\lfloor-\rfloor$ is the rounddown for $\mathbb{Q}$-divisors. That $\mathcal{J}(r \cdot \mathfrak{a})$ is an ideal sheaf follows from the observation that $\mathcal{O}_{Y}\left(K_{Y / X}-\lfloor r E\rfloor\right)$ is a subsheaf of $\mathcal{O}_{Y}\left(K_{Y / X}\right)$ : since $f_{*}\left(\mathcal{O}_{Y}\left(K_{Y / X}\right)\right)=\mathcal{O}_{X}$, $\mathcal{J}(r \cdot \mathfrak{a}) \subset \mathcal{O}_{X}$. We write $\mathcal{J}(\mathfrak{a})$ for $\mathcal{J}(1 \cdot \mathfrak{a})$.

We will now specialize to the case $X=\mathbb{A}^{n}$.

Definition 2. Let $\mathfrak{a} \subset \mathbb{C}\left[x_{1}, \cdots, x_{n}\right]$ be a monomial ideal. We will regard $\mathfrak{a}$ as a subset of the lattice $L=\mathbb{N}^{n}$ of monomials. The Newton polygon $P$ of $\mathfrak{a}$ is the convex hull of this subset of $L$, considered as a subset of $L \otimes \mathbb{R}=\mathbb{R}^{n}$. It is an

Received by the editors April 10, 2000.

2000 Mathematics Subject Classification. Primary 14Q99; Secondary 14M25.

I would like to thank Robert Lazarsfeld for suggesting this problem, and for many valuable discussions. 
unbounded region. $P \cap L$ is the set of monomials in the integral closure of the ideal $\mathfrak{a}$ [7].

Notation 1. We write $\mathbf{1}$ for the vector $(1,1, \ldots, 1)$, which is identified with the monomial $x_{1} x_{2} \ldots x_{n}$. The associated divisor $\operatorname{div}(\mathbf{1})$ is the union of the coordinate axes. We use Greek letters $(\lambda \in L)$ for elements of $L$ or $L \otimes \mathbb{R}$, and exponent notation $x^{\lambda}$ for the associated monomials. For any subset $P$ of $L \otimes \mathbb{R}$, we define $r P$ "pointwise":

$$
r P=\{r \lambda: \lambda \in P\}
$$

We write $\operatorname{Int}(P)$ for the topological interior of $P$, and $\lfloor P\rfloor$ for $\left\{x^{\lfloor\lambda\rfloor}: \lambda \in P\right\}$.

We regard the Newton polygon "officially" as a subset of the real vector space $L \otimes \mathbb{R}=\mathbb{R}^{n}$; the interior operation $\operatorname{Int}(P)$ relies on the real topology of this vector space. However, we don't always carefully distinguish $P$ from the collection of its lattice points $P \cap L$, or from the collection of their associated monomials $\left\{x^{\lambda}: \lambda \in P \cap L\right\}$.

Here is our main result:

Main Theorem. Let $\mathfrak{a} \subset \mathcal{O}_{\mathbb{A}^{n}}$ be a monomial ideal. Let $P$ be its Newton polygon. Then $\mathcal{J}(r \cdot \mathfrak{a})$ is a monomial ideal, and contains exactly the following monomials:

$$
\mathcal{J}(r \cdot \mathfrak{a})=\left\{x^{\lambda}: \lambda+\mathbf{1} \in \operatorname{Int}(r P) \cap L\right\} .
$$

Remark 1. The right hand side, $\left\{x^{\lambda}: \lambda+\mathbf{1} \in \operatorname{Int}(r P) \cap L\right\}$, could instead be called $\lfloor r P\rfloor$. We state the theorem as we do in order to emphasize the monomial $\mathbf{1}$, which is independently important.

Example 1. If $\mathfrak{a}$ is generated by a single monomial, $x^{\lambda}$, then the polygon $P$ is the positive orthant translated upward to $\lambda$, and

$$
\mathcal{J}(\mathfrak{a})=\lfloor P\rfloor=P=\mathfrak{a} .
$$

This is not surprising, because in this case $\mathfrak{a}$ is already a divisor with normal crossing support.

Example 2. Let us calculate the multiplier ideal of $\left(x^{8}, y^{6}\right)$. The Newton polygon is pictured in Figure 1. The distinguished integer vectors $\lambda$ are those with the property that $\lambda+\mathbf{1} \in \operatorname{Int}(P)$. From Figure 1, we conclude that

$$
\mathcal{J}\left(x^{8}, y^{6}\right)=\left(x^{6}, x^{5} y, x^{4} y^{2}, x^{2} y^{3}, x y^{4}, y^{5}\right) \text {. }
$$

Notice that $x^{3} y^{2}$ is almost but not quite in $\mathcal{J}\left(x^{8}, y^{6}\right)$, because $x^{4} y^{3}$ lies on the boundary, not the interior, of the Newton polygon.

Example 3. Let $\left(a_{i}\right)_{i \in n}$ be positive integers, and let $\mathfrak{a}=\left(x_{1}^{a_{1}}, \ldots, x_{n}^{a_{n}}\right)$. One might call this a "diagonal ideal." The only interesting face of the Newton polygon $P$ of $\mathfrak{a}$ is defined by a single dual vector $v=\left(\frac{1}{a_{1}}, \ldots, \frac{1}{a_{n}}\right)$. Therefore $\mathcal{J}(\mathfrak{a})$ contains the monomials $\left\{x^{\lambda}: v \cdot(\lambda+\mathbf{1})>1\right\}$. See [3, example 5.10] for an analytic perspective on this same result. In this expression, the term $v \cdot \mathbf{1}\left(=\frac{1}{a_{1}}+\cdots+\frac{1}{a_{n}}\right)$ may be familiar: It is the log-canonical threshold of $\mathfrak{a}$ (see below).

Example 4. Let $g \in \mathcal{O}_{\mathbb{A}^{n}}$ be an arbitrary polynomial. One might hope that the multiplier ideal associated to the (non-monomial) ideal $(g)$ would be identical to that associated to the monomial ideal $\mathfrak{a}_{g}$ generated by the monomials appearing in $g$. This is not true. Consider $g=(x+y)^{n}$ in $\mathbb{C}[x, y]$. By a linear change of 


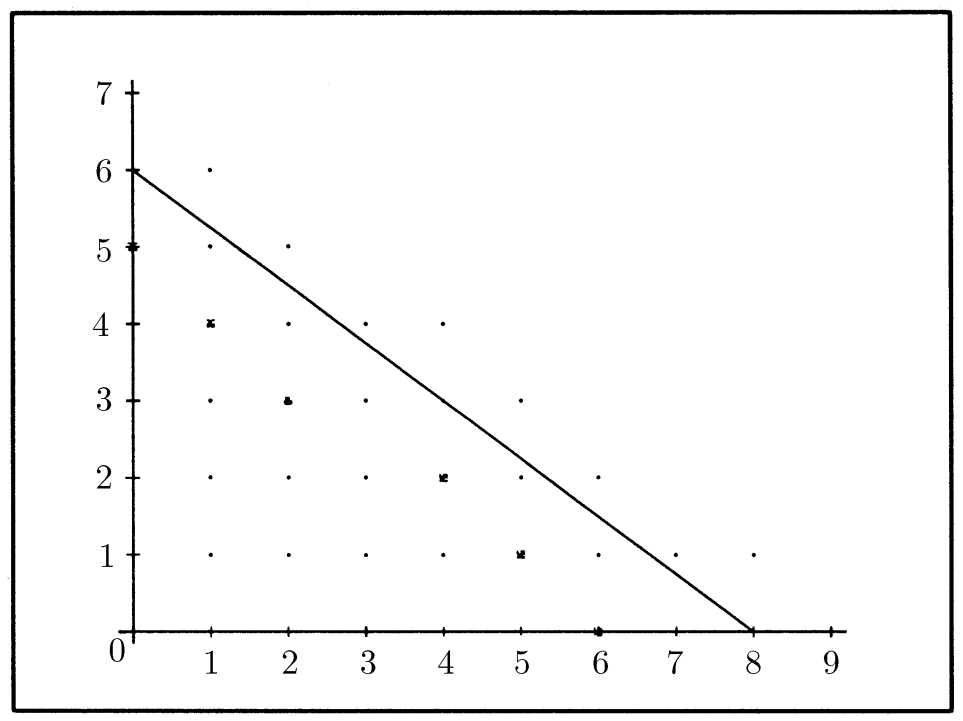

FiguRE 1. The multiplier ideal of $\left(x^{8}, y^{6}\right)$

coordinates in which $z=x+y$ we obtain $g=z^{n}$, and can calculate $\mathcal{J}((g))$ in terms of $z$. This gives $\mathcal{J}((g))=(g) \neq \mathcal{J}\left(\mathfrak{a}_{g}\right)$.

Notice however that for any polynomial $g,(g) \subset \mathfrak{a}_{g}$. It is not difficult to show that $\mathcal{J}(r \cdot(g)) \subset \mathcal{J}\left(r \cdot \mathfrak{a}_{g}\right)$ for all $r$. This containment is almost always strict, but it does become an equality if both $r<1$ and the coefficients of $g$ are sufficiently general.

These conditions guarantee that the multiplicity of the $\mathbb{Q}$ - $\operatorname{divisor} r \cdot \operatorname{Div}(g)$ is less than one away from the zeroes of $\mathfrak{a}_{g}$.

Example 5. Let $\mathfrak{a}$ be a monomial ideal in $\mathbb{A}^{n}$, and let $P$ be its Newton polygon. The $\log$ canonical threshold $t$ of $\mathfrak{a}$ is defined to be

$$
t=\sup \left\{r: \mathcal{J}(r \cdot \mathfrak{a}) \neq \mathcal{O}_{X}\right\} .
$$

See [9] or [4] for a detailed discussion of this concept. The Main Theorem shows that this must be equal to $\sup \{r: \mathbf{1} \notin r P\}$ (provided that $\mathcal{J}(r \cdot \mathfrak{a})$ is nontrivialthe trivial case is an annoying exception). Thus the log canonical threshold is the reciprocal of the (unique) number $m$ such that the boundary of $P$ intersects the diagonal in the $\mathbb{R}^{n}$ at the point $m \mathbf{1}$. In other words, in order to calculate the threshold, we need only find where $P$ intersects the diagonal. Arnold calls this number $m$ of the intersection point the "remoteness" of the polygon. In [2], he proves that $m=\frac{1}{t}$, in order to analyze asymptotic oscillatory integrals.

Example 6. For the "diagonal ideals" of Example 3, the intersection of the diagonal with the Newton polygon is easily calculated using the dual vector $v$. The reader may check that its reciprocal is indeed $v \cdot \mathbf{1}$. (If it happens that $v \cdot \mathbf{1}>1$, then the $\log$ canonical threshold is 1, and the multiplier ideal is trivial.) See 9 for more details. 
Example 7. To illustrate these ideas, we calculate the log-canonical threshold of a slightly more complicated ideal. Let

$$
\mathfrak{a}=\left(x y^{4} z^{6}, x^{5} y, y^{7} z, x^{8} z^{8}\right) .
$$

After drawing the Newton polygon 1 , one sees that the diagonal in $\mathbb{R}^{3}$ intersects the triangular face generated by the first three generators. Therefore, the fourth generator $x^{8} z^{8}$ can be ignored. The intersection of the diagonal with the triangle whose vertices have coordinates $\{(1,4,6),(5,1,0),(0,7,1)\}$ is the point $(m, m, m)$, where $m=\frac{191}{68}$. The $\log$ canonical threshold of $\mathfrak{a}$ is $\frac{1}{m}$, or $\frac{68}{191}$.

The structure of the polygon $P$ can in general be quite complicated, but it must have a single face which intersects the diagonal. This face may not be simplicial, but it certainly decomposes into simplices, one of which intersects the diagonal in the same place and has no more than $n$ vertices. This demonstrates that the log canonical threshold of $\mathfrak{a}$ is equal to that of a smaller ideal generated by no more monomials than the dimension $n$ of the space.

It has been conjectured 2 ([11,, 9$])$ that for every dimension $n$ the collection $\mathcal{T}_{n}$ of all $\log$ canonical thresholds satisfies the Ascending Chain Condition ("All subsets have maximal elements"). The restricted case of ACC for monomial ideals follows from the fact that the partial order of all monomial ideals has no infinite increasing sequences, nor even any infinite antichains [10]. This fact doesn't require any characterization of the thresholds. If ACC is true, then for any fixed dimension $n$, there is a threshold $t_{n}$ closest to, but less than, one. We attempted to use the characterization above to calculate $t_{n}$ in the monomial case, but were unsuccessful. It is known that $t_{1}=1 / 2, t_{2}=5 / 6, t_{3}=41 / 42$, Also, if we restrict to ideals of the form $\mathfrak{a}=\left(x_{1}^{b_{1}}, \ldots, x_{n}^{b_{n}}\right)$, then it is known that we can do no better than $t_{n}=\frac{a_{n}-1}{a_{n}}$, where $a_{1}=2$ and $a_{n+1}=a_{n}^{2}+a_{n}$. The sequence $a_{n}$ is $(2,6,42,1806, \ldots)$. We used a computer to calculate the log canonical threshold for large numbers of monomial ideals, and found no evidence that the above pattern is wrong in general.

\section{Proof of the Theorem}

We will give a straightforward proof of the theorem, based on repeated blowups of the underlying space. The basic proof structure is then an induction, but this creates a problem: After a single such blowup $f: Y \rightarrow X$ the space of interest is no longer $\mathbb{A}^{n}$, so an inductive step doesn't apply.

This difficulty is not a serious one, because $Y$ is still locally $\mathbb{A}^{n}$. Also, all of the above definitions can be extended to $Y$ and onward. For example, the "coordinate axes" on $Y$ should be taken to be proper transforms of those from $X$, together with the exceptional divisor(s). The notion of a "monomial ideal" on $X$ generalizes on $Y$ to an intersection of codimension-1 subschemes (monomials) supported on the "coordinate axes." These extensions are consistent with those obtained by localizing on $Y$ and identifying the coordinate patches with $\mathbb{A}^{n}$ in the obvious way. A briefer argument can be made if one relies on the theory of toric varieties. We will attempt to point out these connections where appropriate.

\footnotetext{
${ }^{1}$ Maple code illustrating this Newton polygon is available from the author by request. Unfortunately, static 2-dimensional representations are not very helpful.

${ }^{2}$ Actually, Shokurov's version of this conjecture is stronger than that presented here. It refers to log canonical thresholds of effective Weil divisors on possibly singular ambient spaces.
} 
Definition 3. By a monomial blowup, we mean a blowup of $X$ along the intersection of some coordinate hyperplanes. By a sequence of monomial blowups, we mean a sequence of blowups, each of which is locally a monomial blowup.

Definition 4. Above we defined 1 as a divisor on $X=\mathbb{A}^{n}$, but we will need a more general notion. If $Y$ is obtained from $X$ by a sequence of monomial blowups, we let $\mathbf{1}_{Y}$ be the divisor which is the sum of the proper transforms of the coordinate axes in $X$, together which each exceptional divisor taken with coefficient 1 . Thus 1 is the union of the "coordinate hyperplanes" of $Y$. We regard $\mathbf{1}_{Y}$ as an element of the lattice $L_{Y}$, which must be defined as the free abelian group on the coordinate hyperplanes in $Y$.

The toric picture better illustrates what's going on here: the exceptional divisors and the proper transforms of the coordinate axes are precisely those effective divisors on $Y$ which are invariant under the natural torus action. Hence $L_{Y}$ is the lattice of torically invariant divisors on $Y$. In general, the sum of all of the effective toric divisors (each with coefficient one) on a toric variety is the anticanonical divisor. So $\mathbf{1}_{X}$ and $\mathbf{1}_{Y}$ are the torically natural anticanonical divisors, and $L_{Y}$ and $L_{X}$ are the lattices of torically invariant divisors.

Lemma 1. Let $X$ be $\mathbb{A}^{n}$ or an intermediate blowup, and let $f: Y \rightarrow X$ be a monomial blowup of $X$. Then

$$
\mathbf{1}_{Y}-f^{*}\left(\mathbf{1}_{X}\right)=K_{Y / X} .
$$

This can be seen without toric geometry by direct calculation; it is easy to pull $\mathbf{1}_{X}$ up to $Y$ and count its multiplicity along the exceptional divisor.

Corollary 1. If $f: Y \rightarrow X$ arises by a sequence of monomial blowups, then

$$
\mathbf{1}_{Y}-f^{*}\left(\mathbf{1}_{X}\right)=K_{Y / X}
$$

The corollary gives a convenient formula for $K_{Y / X}$ when $Y$ is a log-resolution (via a sequence of monomial blowups) of the monomial ideal a. It remains to see that such a space $Y$ exists:

Lemma 2. Let $X=\mathbb{A}^{n}$, and let $\mathfrak{a}$ be a monomial ideal on $X$. Then there is a sequence of monomial blowups $f: Y \rightarrow X$ which constitutes a log-resolution of $\mathfrak{a}$.

Proof. Here we must use some toric geometry. The ideal $\mathfrak{a}$ defines a subset of the lattice $L_{X}$. The dual set of the ideal, $\left\{v \in L_{X}^{*}: \forall \lambda \in P,\langle v, \lambda\rangle \geq 1\right\}$, defines a rational polytope $P^{*}$ in the dual lattice $L_{X}^{*}$. To find a "monomial $\log$ resolution" of $\mathfrak{a}$ is to find a sequence of toric blowups which refine the polytope $P^{*}$ in the appropriate sense. This can be done because $P^{*}$ is rational. The blowups required are exactly those required torically to resolve the singularity of the space $B l_{\mathfrak{a}}(X)$. Figure 2 indicates how this process might be used to resolve the cusp. See [8] section 2.6] for more information on toric resolutions.

We now fix a monomial $\log$-resolution $f: Y \rightarrow X$, as in the lemma. We need to examine the relationship between $\mathfrak{a}$ and $f^{-1}(\mathfrak{a})$. By the definition of $f, f^{-1}(\mathfrak{a})$ is a line bundle. It corresponds to a divisor whose support is contained in the proper transforms of the coordinate axes from $X$ and the exceptional divisors. We called the collection of such divisors $L_{Y}$. To $f^{-1}(\mathfrak{a})$ we may associate a single element $\gamma$ of $L_{Y}$, its "generator." We may even give it a Newton polygon $P_{Y}$, namely the positive orthant translated to $\gamma$. 


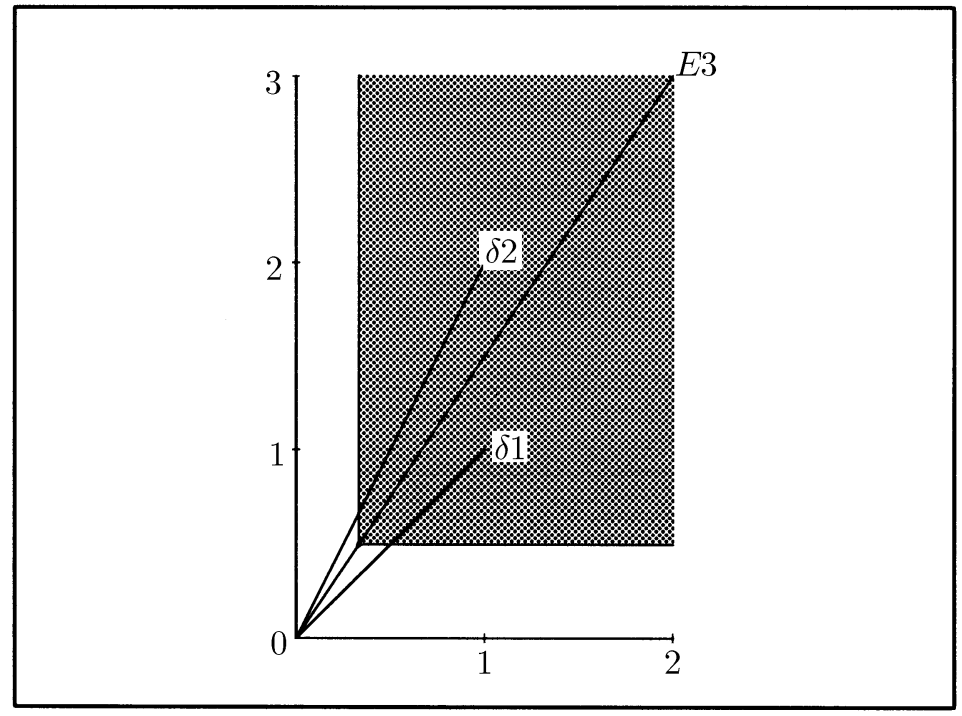

Figure 2. The resolution of the cusp ideal $\left(x^{3}, y^{2}\right)$ via dual polytope refinement. The large rectangle is the dual polytope $P^{*}$ for this ideal. The cusp is fully resolved because the ray representing $E_{3}$ contains the lower left corner of the dual polytope, splitting it apart into sections without corners.

Lemma 3. Let $f: Y \rightarrow X$ resolve $\mathfrak{a}$ by a sequence of monomial blowups. Let $P_{X}$ be the Newton polygon of $\mathfrak{a}$, and let $P_{Y}$ be as above. Since $f^{*}$ acts linearly on the lattices, we may extend it to all of $L_{X} \otimes \mathbb{R}$. When we do this,

1. $f^{*}$ takes the interior points of $P_{X}$ to interior points of $P_{Y}$.

2. $f^{*}$ takes the boundary points of $P_{X}$ to boundary points of $P_{Y}$.

3. $f^{*}$ takes the points not in $P_{X}$ to points not in $P_{Y}$.

Proof. The lemma hinges on three basic ideas. First, $f^{*}$ is certainly a map from $L_{X}$ to $L_{Y}$, but because it is linear, $f^{*}$ extends to all of $L_{X} \otimes \mathbb{R}$ in a natural way. As a map of real vector spaces, $f^{*}$ is continuous because it is linear. Second, for each of the effective toric divisors, or "coordinate planes" $E_{i}$ in $Y$,

$$
\operatorname{ord}_{E_{i}}\left(f^{*}\left(\mathbf{1}_{X}\right)\right)>0 \text {. }
$$

The equality is strict because the blowups permitted are monomial. Third, we have the standard equation $f_{*}\left(\mathcal{O}_{Y}(-E)\right)=\overline{\mathfrak{a}}$, where $\overline{\mathfrak{a}}$ is the integral closure of $\mathfrak{a}$.

We will prove the lemma by proving part 3 first for integral points $\lambda \in L_{X} \otimes \mathbb{R}$, then for rational points, and finally for real points. We will prove part 1 by using the strict positivity of $f^{*}\left(\mathbf{1}_{X}\right)$. Finally, we'll deduce part 2 by continuity.

Let $\lambda$ be an integer point of $L_{X}$ not in $P_{X}$. Then $x^{\lambda} \notin \overline{\mathfrak{a}}=f_{*}\left(\mathcal{O}_{Y}(-E)\right)$, so $f^{*}(\lambda) \notin P_{Y}$. If instead $\lambda \notin P_{X}$ has rational coordinates, then we can clear denominators. Let $n \lambda$ be integral. $n \lambda \notin n P(\mathfrak{a})=P\left(\mathfrak{a}^{n}\right)$, so $f^{*}(n \lambda) \notin f^{*}\left(\mathfrak{a}^{n}\right)=$ $n P_{Y}$. (Here we have used the just-proved integer case, as well as the fact that the resolution $f: Y \rightarrow X$ resolving $\mathfrak{a}$ also resolves $\mathfrak{a}^{n}$.) Dividing by $n$ gives $f^{*}(\lambda) \notin P_{Y}$. If $\lambda \notin P_{X}$ has real coordinates, choose a rational $\mu \geq \lambda$ also not in $P_{X}$. Then $f^{*}(\lambda) \leq f^{*}(\mu) \notin P_{Y}$, so $f^{*}(\lambda) \notin P_{Y}$. 
A standard convexity argument proves that if $\lambda \in P_{X}$, then $f^{*}(\lambda) \in P_{Y}$. To prove part 1 of the lemma, let $\lambda$ be in the interior of $P_{X}$. Choose $\mu \in P_{X}$ and $\epsilon \in \mathbb{R}^{+}$with $\lambda=\mu+\epsilon \mathbf{1}$. Then $f^{*}(\lambda)=f^{*}(\mu)+\epsilon f^{*}(\mathbf{1})$. Also, $f^{*}(\mu) \in P_{Y}$, and $\epsilon f^{*}(\mathbf{1})$ is strictly positive in every coordinate, so $f^{*}(\lambda)$ is in the interior of $P_{Y}$.

Part 2 of the lemma follows from the continuity of the map $f^{*}$.

We can now give the proof of the main theorem. Because $\mathcal{J}(r \cdot \mathfrak{a})$ is invariant under the natural torus action, it must be a monomial ideal. We characterize the monomials $x^{\lambda}$ in $\mathcal{J}(r \cdot \mathfrak{a})$. By definition, $x^{\lambda}$ is in $\mathcal{J}(r \cdot \mathfrak{a})$ if and only if

$$
\operatorname{div}\left(f^{*}\left(x^{\lambda}\right)\right)+K_{Y / X}-\lfloor r E\rfloor \geq 0
$$

(recall that $\left.\mathcal{O}_{Y}(-E)=f^{-1}(\mathfrak{a})\right)$. This condition simply means that

$$
\operatorname{div}\left(f^{*}\left(x^{\lambda}\right)\right)+K_{Y / X} \text { is in }\left\lfloor r P_{Y}\right\rfloor
$$

(also recall that $\left\lfloor r P_{Y}\right\rfloor=\left\{x^{\lfloor\lambda\rfloor}: \lambda \in r P\right\}$ ). Using the calculation of $K_{Y / X}$ from Lemma 1, this can be rewritten

$$
\operatorname{div}\left(f^{*}\left(x^{\lambda}\right)\right)-\mathbf{1}_{Y}+f^{*}\left(\mathbf{1}_{X}\right) \in\left\lfloor r P_{Y}\right\rfloor .
$$

This is of the form $\{$ divisor $\}-\mathbf{1}_{Y} \in\left\lfloor r P_{Y}\right\rfloor$, so we rewrite it as $\{$ divisor $\} \in \operatorname{int}\left(r P_{Y}\right)$, obtaining

$$
\operatorname{div}\left(f^{*}\left(x^{\lambda}\right)\right)+f^{*}\left(\mathbf{1}_{X}\right) \in \operatorname{int}\left(r P_{Y}\right) .
$$

But this is just a condition on divisors from $X$. By Lemma 3 parts 1 and 2 , it is equivalent to $\left(\lambda+\mathbf{1}_{X}\right) \in \operatorname{Int}\left(r P_{X}\right)$. The theorem is proved.

\section{REFERENCES}

[1] Urban Angehrn and Yum Tong Siu. Effective freeness and point separation for adjoint bundles. Invent. Math., 122(2):291-308, 1995. MR 97b:32036

[2] V. I. Arnol'd, S. M. Guseřn-Zade, and A. N. Varchenko. Singularities of Differentiable Maps. Vol. I. Birkhäuser Boston Inc., Boston, Mass., 1985. MR 86f:58018

[3] Jean-Pierre Demailly. $L^{2}$ vanishing theorems for positive line bundles and adjunction theory. In Transcendental methods in algebraic geometry (Cetraro, 1994), Lecture Notes in Math., vol. 1646, pages 1-97. Springer, Berlin, 1996. MR 99k:32051

[4] Jean-Pierre Demailly and János Kollár. Semi-continuity of complex singularity exponents and Kähler-Einstein metrics on Fano orbifolds. Mathematics E-Print Archive, October 1999.

[5] Lawrence Ein. Multiplier ideals, vanishing theorems and applications. In Algebraic geometry - Santa Cruz 1995, Proc. Sympos. Pure Math., vol. 62, part 1, pages 203-219. Amer. Math. Soc., Providence, RI, 1997. MR 98m:14006

[6] Lawrence Ein and Robert Lazarsfeld. A geometric effective Nullstellensatz. Invent. Math., 137(2):427-448, 1999. MR 2000j:14028

[7] David Eisenbud. Commutative Algebra. With a View toward Algebraic Geometry. SpringerVerlag, New York, 1995. MR 87a:13001

[8] William Fulton. Introduction to Toric Varieties. Princeton University Press, Princeton, NJ, 1993. MR 94g:14028

[9] János Kollár. Singularities of pairs. In Algebraic geometry - Santa Cruz 1995, pages 221-287. Proc. Sympos. Pure Math., vol. 62, part 1, Amer. Math. Soc., Providence, RI, 1997. MR 99m:14033

[10] Maclagan. Antichains of monomial ideals are finite. Mathematics E-Print Archive, October 1999.

[11] V. V. Shokurov. 3-fold log flips. Izv. Ross. Akad. Nauk Ser. Mat., 56:105-203, 1992; English transl., Russian Acad. Sci. Izv. Math. 40:95-202, 1993. MR 93j:14012

[12] Yum-Tong Siu. Invariance of plurigenera. Invent. Math., 134(3):661-673, 1998. MR 99i:32035

Department of Mathematics, University of Michigan, Ann Arbor, Michigan 48104

E-mail address: jahowald@math.1sa.umich.edu 